\title{
Corela
}

Cognition, représentation, langage

HS-31 | 2020

Métalinguistiques.

\section{Commentaire métalinguistique et partialité du dire : enjeux de la classification opérationnelle de quelques marqueurs discursifs en allemand}

Pierre-Yves Modicom

\section{OpenEdition}

Journals

Édition électronique

URL : http://journals.openedition.org/corela/11266

DOI : $10.4000 /$ corela. 11266

ISSN : 1638-573X

Éditeur

Cercle linguistique du Centre et de l'Ouest - CerLICO

Référence électronique

Pierre-Yves Modicom, «Commentaire métalinguistique et partialité du dire : enjeux de la classification opérationnelle de quelques marqueurs discursifs en allemand », Corela [En ligne], HS-31 | 2020, mis en ligne le 02 juillet 2020, consulté le 03 juillet 2020. URL : http://journals.openedition.org/corela/11266 ; DOl : https://doi.org/10.4000/corela.11266

Ce document a été généré automatiquement le 3 juillet 2020

\section{(c) (i) (2)(2)}

Corela - cognition, représentation, langage est mis à disposition selon les termes de la licence Creative Commons Attribution - Pas d'Utilisation Commerciale - Partage dans les Mêmes Conditions 4.0 International. 


\title{
Commentaire métalinguistique et partialité du dire : enjeux de la classification opérationnelle de quelques marqueurs discursifs en allemand
}

\author{
Pierre-Yves Modicom
}

1 En quoi peut-on distinguer une notion spécifique de « commentaire métalinguistique » au sein des opérations énonciatives? Si l'on aborde cette question sous l'angle des marqueurs discursifs (MD), on rencontre souvent des classifications posant une différence nette entre les opérations de reformulation et/ou de commentaire métalinguistique d'une part, et d'autre part des opérations discursives de modalisation du propos, ou de pondération entre points de vue (Breindl et al. 2014 ; Paillard 2009). Cet article adopte une conception de l'énonciation accordant une place centrale aux tâtonnements et à la réélaboration du propos; nous verrons que cela nous conduit à traiter le commentaire métalinguistique comme un simple sous-type d'une opération plus générale d'ostension de la réélaboration. Pour ce faire, nous partirons d'un problème de position des MD dans l'énoncé assertif en allemand pour construire une classe de «mots du discours de point de vue » (Paillard 2009) dont nous verrons dans un second temps qu'elle doit inclure les marqueurs de reformulation.

\section{L'« après-première place » de l'énoncé assertif allemand}

2 En allemand, l'énoncé assertif se caractérise par une règle V2 pour « verbe en deuxième position ", c'est-à-dire que la forme personnelle du verbe y est obligatoirement précédée d'un et un seul syntagme. 
(1a) Ich weiß nichts davon.

'Je n'en sais rien.'

(1b) Davon weiß ich nichts.

(1c) *Weiß ich nichts davon.

(1d) *Ich davon weiß nichts.

(1e) * Davon ich weiß nichts.

3 Il existe deux exceptions à cette règle : (i) dans quelques types d'énoncés fortement stéréotypés et/ou associés à un genre de discours particulier (débuts de récits), la forme personnelle $\mathrm{du}$ verbe est en première position ; (ii) il existe une construction ${ }^{1}$ dans laquelle un MD s'insère entre le constituant initial et la forme personnelle du verbe. Le MD est alors dit occuper l'après-première place (APP) de l'énoncé. Cette construction fournira le point de départ de notre étude. D’après Breindl $(2008,2011)$, l'APP est sujette à deux contraintes majeures :

4 - le premier constituant de l'énoncé est obligatoirement un topic contrastif ${ }^{2}$

5 - le constituant en APP est alors obligatoirement un marqueur discursif, sans que tous les MD soient pour autant licites dans cette construction.

(2) Warum gibt es denn die große Pleitewelle in Bayern ? Ich behaupte nicht, dass das die Schuld von Edmund Stoiber ist. Vielmehr liegt es daran, dass in Bayern besonders viele Unternehmen des Neuen Marktes - da gab es ja fantastische Vorstellungen - beheimatet waren.

Kirch allerdings gehörte nicht zum Neuen Markt, sondern in diesem Fall waren das wollen wir nicht vergessen - die Bayerische Landesbank und das bayerische Kabinett die Verantwortlichen. (DEREKO- REI/BNG.00174 - Joschka Fischer, 04.07.2002)

'Pourquoi cette grande vague de faillites en Bavière ? Je ne prétends pas que c'est la faute d'Edmund Stoiber. C'est plutôt lié au fait que s'y trouvaient un nombre particulièrement important $d$ 'entreprises du nouveau marché - où on se faisait des idées à un point incroyable.

Toutefois, Kirch, lui, ne faisait pas partie du nouveau marché, mais dans ce cas-là, et nous ne devons pas l'oublier, c'étaient la banque régionale bavaroise et le gouvernement bavarois les responsables.'

6 A l'oral, on aura :

(2') /KIRCH allerdings [.] gehörte /NICHT \zum Neuen Markt.

7 Bien qu'allerdings soit parfois considéré comme un " connecteur adversatif » au même titre que trotzdem (litt. " malgré cela », cf. la classification de Breindl et al. (2014)), ce dernier n'est pas licite en APP.

(3) *Kirch trotzdem gehörte nicht zum Neuen Markt.

8 Les connecteurs « au sens strict » (« vrais connecteurs » de Nølke (1993), notamment tous ceux pour lesquels la tradition grammaticographique allemande parle «d'adverbes conjonctionnels », Konjunktionaladverbien, dont la morphologie inclut une préposition et un morphème anaphorique) ne sont jamais licites en APP (cf. p.ex. deswegen, deshalb, daher pour la cause). Indépendamment de l'insuffisance du concept de connecteur lorsqu'il s'agit de décrire des MD participant de la cohésion textuelle (cf. Paillard (2009)), ce n'est donc pas la « connectivité » qui est ici en jeu. Dalmas (1995) parle à leur propose de « pseudo-connecteurs ».

9 À cela s'ajoute une propriété que Breindl ne relève pas : la polarité positive forte. Tous les MD licites en APP sont bloqués dans les énoncés interrogatifs autres que rhétoriques et après la négation; quand ils apparaissent dans une subordonnée hypothétique, ils 
prennent portée par-dessus le subordonnant, sur l'ensemble subordonnée + principale. On peut illustrer cette sensibilité à la polarité en opposant allerdings et trotzdem dans les subordonnées hypothétiques.

(4) Zweitens halte ich es nicht für wikipedida-relevant (wenngleich ich es interessant finde). Außerdem sind die Schlussfolgerungen, die gezogen werden, bezweifelbar. Wenn du trotzdem etwas schreiben willst, nur zu, andere werden es bestimmt verbessern, wenn's nicht ganz so toll aussieht. (DEREKO - WDD11/ A06.57387)

'Deuzio, je ne pense pas que ce soit pertinent pour wikipedia, même si je trouve ça intéressant en soi. En outre, les conclusions tirées sont discutables. Mais si tu veux quand même écrire quelque chose, vas-y, d'autres ne manqueront pas d'apporter des corrections si le résultat n'est pas extraordinaire.'

10 Trotzdem reste dans la portée de l'opérateur hypothétique : c'est le contenu de la protase qui est pris dans une contradiction avec le cotexte gauche, qui livre des arguments pour considérer cette hypothèse comme incongrue.

(5) Die Dinge, die ich nicht bearbeitet habe - ich glaube, es sind nicht viele - habe ich mit gutem Grund so gelassen, weil ich sie für richtig erachte. Sollte ein anderer jedoch nicht meiner Meinung sein, ist er herzlich eingeladen, zu verbessern. Wenn allerdings ein großer Abschnitt komplett bearbeitet werden soll, wäre ich dem Nutzer sehr verbunden, wenn er mich vorher kontaktieren könnte. (DEREKO WDD11/A22.01205:)

‘Tout ce que je n'ai pas modifié (je crois que ça ne représente pas grandchose), je l'ai laissé en l'état pour de bonnes raisons, vu que je considérais que c'était exact. Si toutefois quelqu'un n'était pas d'accord, il est cordialement invité à apporter ses améliorations. Par contre, si c'est une grande section qui doit être complètement refondue, je serais très reconnaissant à cet utilisateur de me contacter auparavant.'

11 Allerdings prend portée sur l'ensemble de l'énoncé ; la protase correspond à un cadre hypothétique (au sens de Charolles (1997)) qui s'oppose au monde précédemment construit, et s'il y a une tension logique avec le cotexte gauche, elle concerne surtout l'apodose, puisque l'on aurait effectivement pu envisager que le locuteur ne voie pas d'un bon œil les avis divergents. Le français permet de rendre cette relation de portée en isolant par contre en tête d'énoncé. Face à ces différences, il nous semble donc nécessaire de reprendre la liste des domaines sémantiques dont relèvent les MD concernés telle que la donne Breindl sans la commenter.

\section{Inventaire sémantique des formes licites en APP :}

12 - adversativité / additivité : allerdings, jedoch (« pourtant »), nun (« or »), jedenfalls (« en tout cas $»), \ldots$

- modalisation: adverbes modaux épistémiques (wahrscheinlich, vermutlich "probablement », ...) et axiologiques (leider « malheureusement », hoffentlich «il est à espérer que »)

- ordre de réalité / de vérité : in Wahrheit (« en vérité »), in Wirklichkeit (« en réalité »), eigentlich ( en réalité »).

- structuration textuelle «pure », dont les marqueurs d'intégration linéaire (Auchlin 1981, Turco \& Coltier 1988) : einerseits / andererseits (« d'une part / d'autre part »), schlie 
Blich (« enfin »), ...

- exemplification (beispielsweise, zum Beispiel)

- reformulation et commentaire métalinguistique (cf. infra)

$13 \mathrm{Au}$ vu de cet inventaire, nous suggérons que ces marqueurs participent tous, plus que de la cohésion textuelle, d'un métacommentaire portant sur le dire lui-même.

\section{Partialité du dire}

14 Ce qui est mis en jeu dans le métacommentaire dont participent les MD licites en APP nous semble être ce que Paillard appelle la « partialité du dire » (Paillard 2009), c'est-àdire le fait qu'une assertion est une façon partielle et partiale de dire un état de chose. Pour mieux appréhender l'APP, nous tenterons d'articuler cette notion de partialité du dire à des concepts de l'étude de la structure informationnelle de l'énoncé, attendu que le constituant initial d'un énoncé avec MD en APP est obligatoirement un "topic contrastif ». Nous entendons l'expression « topic contrastif » au sens de l'aboutness shift topic de Frascarelli \& Hinterhölzl (2007) (cf. Breindl (2011) et Büring (2003)). Le topic contrastif permet de construire le domaine de référence d'un ou plusieurs énoncés en l'opposant à un autre domaine de référence plausible / accessible en contexte. Pour Féry (2007), cet " autre » sous-déterminé vis-à-vis duquel il y aurait « contraste » est tantôt un ensemble hypéronymique dont le topic contrastif serait un sous-ensemble, tantôt comme un autre sous-ensemble au sein d'un même ensemble. Ainsi, dans l'exemple (2), Kirch, qui désigne un groupe de télévision allemand ayant fait faillite, s'oppose aux «nombreuses entreprises du Nouveau Marché » mentionnées dans l'énoncé précédent, au sein d'un ensemble plus vaste, celui des entreprises bavaroises ayant fait faillite dans les années précédant la déclaration de Joschka Fischer. Sur ce point, on peut faire le parallèle avec les "cadres" de Charolles (1997): le topic contrastif a pour fonction de délimiter des segments textuels à une échelle qui n'est pas celle de l'énoncé mais plutôt de la séquence, en introduisant des solutions de continuité dans le texte ; en ce sens, loin de la " connexion", un énoncé avec constituant en APP instaure plutôt une rupture discursive avec ce qui précède. Plus généralement, le topic, compris comme " ce dont on parle ", est le signe d'un dire partiel, sélectif dans ce dont il parle, et incapable d'épuiser tout ce qu'il y aurait à dire.

Mais en fait d'« un » topic, les travaux sur la structure de l'énoncé allemand ${ }^{3}$ ont bien montré qu'on avait plutôt affaire à un domaine de constituants topicaux (on préférera du coup, à la suite de Zemb (1978), l'adjectif thématique), que l'on peut concevoir comme les traces d'autant d'opérations successives de repérage. Le constat est vrai également dans la perspective de Charolles (1997), où les cadres référentiels, les seuls qui soient topicaux pour Strawson (1971) ou Reinhardt (1981), coexistent avec des cadres spatiaux, temporels, etc. Au sein de ce domaine des constituants thématiques, l'allemand oblige à singulariser un syntagme qui sera placé en tête d'énoncé, et qui peut faire l'objet d'une mise en contraste telle que l'on vient de la décrire.

Le premier constituant revêt la valeur de point d'amorce de l'énoncé dans son ensemble (Dalmas 2011). Dans la perspective de la "partialité du dire", cela nous conduit à émettre l'hypothèse que dès lors qu'un énoncé est partiel et partial, le premier constituant donne à voir le "point de perspective » dont l'énoncé est représentatif. Signalons enfin que les autres exceptions à la règle V2, c'est-à-dire les énoncés assertifs débutant directement par la forme verbale, sont justement des 
énoncés thétiques. Ces énoncés se présentent comme des jugements épuisant ce qu'il y a à dire (on postule d'ordinaire que la question à laquelle ils apportent une réponse est «qu'y a-t-il? ? et que s'il fallait leur trouver un thème, ce serait «ce qui est » en général).

Ce faisceau d'indices nous conduit à l'idée que le constituant thématique initial définit en quoi un énoncé est partiel, c'est-à-dire marque la discrimination entre ce dont on parle et ce dont on ne parlera pas. Cette partialité peut n'être que latente, ne donner lieu à aucun marquage "méta ». C'est le cas le plus fréquent. Le marquage prosodique (accent de hauteur à profil ascendant) ou syntaxique (détachement à gauche) ${ }^{4}$ représente un premier mouvement de bouclage, la partialité constitutive devenant une partialité explicite, "montrée" au sens où Authier-Revuz (1984) opposait le « constitutif » et le " montré » dans sa réflexion sur l'hétérogénéité énonciative. L'étape suivante est la mise en œuvre d'un « méta » explicite, avec le MD en APP : le dire y cesse de se donner comme simplement partiel et peut maintenant être vu comme partial, le MD étant l'indice du biais caractérisant le point de vue choisi. Le marquage prosodique du topic contrastif est une condition à l'apparition du MD en APP : en faisant basculer la partialité du constitutif-latent vers le montré, il ouvre le créneau pour la spécification additionnelle du biais.

Le fait qu'allerdings soit au départ un adverbe affirmant la validité d'un jugement « en tout état de cause" (l'étymologie est parfaitement transparente en allemand contemporain) pose d'ordinaire bien des problèmes à la description (cf. Métrich et al. (1998: 198 sq.) ; une exception notable : Pérennec (2002: 125-141)). La valeur oppositive adventice s'est développée en discours: allerdings présente l'énoncé comme représentatif d'un point de vue général, englobant et immunisé contre les objections de détail (le propos est valide "quelles que soient les prémisses avancées en amont »). On retrouve le mécanisme décrit par Paillard: la caractérisation de l'énoncé comme représentatif de tel ou tel type de perspective implique en creux qu'un énoncé " autre ", typiquement l'énoncé précédent, soit dépourvu de la qualité qui a présidé à l'élaboration du dire finalement réalisé ; en l'espèce, il s'agit de présenter l'affirmation selon laquelle les contre-performances bavaroises sont imputables à la crise générale du Nouveau Marché comme une vérité parcellaire qui vient se heurter au contreexemple de Kirch, lequel est lui-même pourvu d'une valeur de généralité supérieure qui vient bien sûr servir l'argumentation.

\section{3. « Mots du dire »}

Venons-en maintenant au cas particulier de ces marqueurs métalinguistiques qu'à la suite de Paillard (2009) et Khatchatourian (2008) nous qualifions de "mots du dire». Comme en français, il s'agit de formes figées à partir d'une tournure impliquant un verbe comme dire. Typiquement, il s'agira du participe accompli des verbes sagen ( dire ») ou formulieren ( formuler ») qualifié par un adjectif antéposé (les adjectifs en allemand peuvent accéder aux fonctions adverbiales). On obtient des formes comme ehrlich gesagt (litt. « sincèrement dit »), kurz gesagt (litt. « brièvement dit »), besser gesagt ( mieux dit»), anders formuliert («formulé autrement»). Deux remarques s'imposent ici. La connectivité éventuelle de ces formes ne semble pas être tant question de leur appartenance à la classe des mots du dire que du signifié de l'adjectif et/ou de son 
degré : un adjectif syncatégorématique comme autre ou une forme au comparatif induisent une connectivité plus forte, ou plus immédiate.

Ces formes s'opposent, sur le plan syntaxique, à un autre groupe de marqueurs métalinguistiques qui se construisent comme des conjonctions de coordination et non comme des syntagmes adverbiaux : $:^{5}$ das heißt («c'est-à-dire", marqueur de reprise selon Murat \& Cartier-Bresson (1987)), beziehungsweise (litt. "relationnellement» > « et », « ou », " respectivement »). On verra à l'occasion que pour ce qui est des valeurs discursives, das heißt « c'est-à-dire » ne s'oppose pas fondamentalement à anders gesagt « autrement dit ».

(6) Das hat dazu geführt, dass in vielen Schulen die Lernziele modularisiert werden, das heißt, dass es Aufgabenbereiche gibt, die sich Schüler nacheinander vornehmen, wo sie aber zu unterschiedlichen Zeiten dran arbeiten. (DR 170913-01)

Cela a conduit à ce que dans beaucoup d'écoles, les objectifs pédagogiques sont modularisés, c'est-à-dire qu'il y a des domaines de travail que les élèves étudient successivement mais qu'ils y travaillent à des moments différents.

(7) Natürlich lügen die Bilder, das heißt, eigentlich lügen sie gar nicht. (internet) ${ }^{6}$ 'Bien sûr les images nous mentent, c'est-à-dire qu'en fait elles ne nous mentent absolument pas.'

21 Les mots du dire, contrairement à ces vrais-faux « coordonnants ", semblent licites en APP, mais apparaissent plutôt en incise.

(8) Viele PDS-Wähler konnten aus dem Reservoir früherer Nicht-Wähler mobilisiert werden. Nicht zuletzt der PDS, anders gesagt, ist die gestiegene Wahlbeteiligung zu verdanken. (cit. GrWB, article anders gesagt)

'Beaucoup d'électeurs du PDS ont été gagnés sur le réservoir des anciens abstentionnistes. En d'autres termes, c'est en bonne partie au PDS que l'on doit la participation en hausse.'

(9) Fast täglich treffen ihn die Ärzte, Sekretärinnen und seine ehemaligen Mitarbeiter in der Abteilung. Sein Büro genau gesagt lässt ihn nicht los. (op.cit., article genau gesagt)

'Presque tous les jours, les médecins, secrétaires et anciens collaborateurs le croisent dans le service. Son bureau, plus précisément, ne le lâche pas.'

Que faire de cette préférence pour l'incise, qui distingue les mots du dire des autres MD licites en APP, qui sont davantage insérés dans la structure prosodique de l'énoncé à l'oral et, par ricochet, très peu écrits entre virgules lorsque le support de l'énoncé est graphique? Il convient de relever que les "vrais» connecteurs comme trotzdem ou deshalb ne sont pas plus licites en APP en incise que lorsque l'on tente de les intégrer prosodiquement. Rappelons-nous l'exemple (2) :

(2) Kirch allerdings gehörte nicht zum Neuen Markt.

'Cela dit, Kirch, lui, ne faisait pas partie du nouveau marché.'

23 On ne peut pas utiliser trotzdem à la place d'allerdings (ex. 3, cf. plus haut), et il en va de même en incise (ex. $\left.3^{\prime}\right)$.

(3') ??? Kirch - trotzdem - gehörte nicht zum Neuen Markt.

24 Par conséquent et comme le proposent Breindl (2011) et le dictionnaire grammatical (GrWB) de l'Institut pour la langue allemande IDS, les mots du dire de l'allemand 
peuvent être maintenus dans la catégorie des MD licites en APP, par opposition à ceux que l'on ne trouve jamais dans cette position et dont font partie tous les véritables connecteurs. Faute de meilleure explication, nous proposons de considérer l'intégrabilité déficiente de ces formes par leur caractère en partie idiomatique, c'est-àdire le fait qu'il s'agisse d'unités polylexématiques stables (UPS, Espinat (2015)), ladite stabilité étant justement encore relative, puisque le flottement entre gesagt et formuliert, la compositionnalité stricte du sens, la gradabilité des adjectifs employés et la transparence absolue des expressions peuvent être pris comme autant de signes d'un figement qui n'est encore que partiel. Les structures en gesagt ou formuliert sont sans doute encore interprétées comme des syntagmes complexes et non comme des « adverbes » pleinement lexicalisés.

Dans ces conditions, il semble plausible de traiter les mots du dire comme les marqueurs d'un type particulier de partialité énonciative. De fait, dans la mesure où cette notion est fortement tributaire d'une conception de l'énonciation comme une élaboration progressive, tâtonnante et cumulative, c'est-à-dire en somme travaillée par une reformulativité inhérente, pourquoi ne pas considérer les marqueurs participant le plus ouvertement de la reformulation comme un cas extrême ? De même qu'il y a une partialité constitutive-latente et une partialité montrée, il pourrait y avoir, toujours dans le sillage des propositions d'Authier-Revuz (1984) sur l'hétérogénéité énonciative, une reformulativité constitutive distincte de la reformulativité montrée. Reste que dans la typologie de Paillard (2009), les mots du dire ne sont pas un type de «mots du discours de point de vue ", ces derniers ayant l'apanage du marquage de la partialité du dire. D'ailleurs, il n'est pas sûr que bref ou franchement seraient considérés par lui ni comme des «mots du dire » ni comme des «mots du discours de point de vue ». Qu'en est-il alors à la fois du rapport entre mots du dire et partialité et du rapport entre reformulativité constitutive et reformulativité montrée?

Nous pouvons distinguer différents (sous-)groupes de mots du dire en allemand, selon le type de partialité qu'ils mettent en jeu, et qu'on ne peut réduire à une noncoïncidence du dit au vouloir-dire.

27 A. Relation sous-spécifiée d'équivalence au niveau du vouloir-dire : anders gesagt, anders formuliert, mit anderen Worten (« en d'autres termes »)

28 B. Formes spécialisées dans un certain type d'ajustement : (cf. emploi fréquent du comparatif en -er)

29 (Non-)coïncidence des mots aux choses :

1. rectification : besser gesagt ( mieux »), genau gesagt (" précisément »), genauer gesagt 2. approximation: pauschal gesagt (" à l'emporte-pièce"), grob gesagt (" grossièrement »), überspitzt formuliert (« de façon exagérée »)

(Non-)coïncidence des mots aux conditions de l'échange:

3. efficacité : kurz gesagt («brièvement»), kürzer gesagt, knapp formuliert (« en peu de mots »), kurzum («bref»)

30 4. intelligibilité $:$ einfach gesagt $($ einfach $=$ " simple»), vereinfacht gesagt $($ vereinfacht $=$ « simplifié »)

31 Notre propos ici n'est pas de nous attarder sur cette classification, qui du reste et en tout cas pour le groupe B est inférable de la sémantique individuelle de l'adjectif construit avec gesagt ou formuliert. Nous reviendrons plutôt sur le type A, celui des marqueurs de type autrement dit / en d'autres termes, qui à première vue semblent se 
situer le plus directement et le plus «simplement» dans le registre d'une paraphrase prétendument neutre sur le plan du contenu, d'une variation « de forme » sans toucher au «fond». Il n'en est rien, et l'hétérogénéité mise en jeu par la réélaboration du propos nous semble procéder à un changement de point de vue au sens entendu plus haut, c'est-à-dire que le métalinguistique fait retour vers ce que le propos tente de saisir. Pour illustrer notre propos, prenons ici deux exemples.

(10) Es gab damals aber auch - und das war nicht gut - sehr viel wirkliches Misstrauen zwischen dem, was damals die CDU die Sozis nannte, die nicht mit Geld umgehen konnten, und umgekehrt die Vorwürfe. Mit anderen Worten : Da gab es auch eine Polarisierung der politischen Kultur und ein Misstrauen in den beiden Mehrheitslagern, das auch für eine demokratische Gesellschaft, wenn es einen bestimmten Punkt überschreitet, nicht gut ist. (DR 200913-07)

'Mais à l'époque il y avait aussi - et ça ça n'était pas bon - une très grande méfiance entre ce (sic) que la CDU appelait alors « les socialos qui ne savent pas s'occuper d'argent ", et réciproquement. En d'autres termes : il y avait à l'époque une polarisation de la culture politique et une méfiance dans les deux camps majoritaires qui n'est pas bonne non plus dans une société démocratique lorsqu'elle dépasse un certain stade.'

La construction d'une relation d'identification permet de passer d'un propos particulier à un point de vue général, dans un mouvement converse de celui opéré par les marqueurs d'exemplification, mais qui peut également être rapproché d'une consécution logique. Prenons un exemple, tiré de l'interview d'un "spécialiste des médias» à propos du $25^{\mathrm{e}}$ anniversaire d'une prise d'otage de plusieurs jours hypermédiatisée dans la localité allemande de Gladbeck. Dans ce passage, il est question de ce qui distingue cet événement paroxystique d'autres épisodes de sensationnalisme journalistique.

(11) Bei Gladbeck kam hinzu, dass es auf der einen Seite aus den genannten Gründen diesen Reflex des realen eines realen Geschehens gab, auf der anderen Seite aber Gladbeck speziell so etwas - so pervers das klingen mag - wie eine Story mit Cliffhangern war. Das heißt, das Geschehen lief über mehrere Tage und keiner, nicht mal die Täter, keiner wusste ja, wie es ausgeht. Und mit anderen Worten, das war natürlich so wie so ungefähr der ultimative Kick, die ultimative, ultimative Geschichte, kein Mensch wusste, wie es ausging, und jeder war natürlich gespannt, gehts gut aus, gehts schlecht aus? (DR 160813-04)

'Dans le cas de Gladbeck, il faut ajouter à cela qu'il y avait d'une part pour les raisons déjà citées le réflexe de l'événement réel, d'un événement réel, et que de l'autre côté par contre Gladbeck en particulier était quelque chose, même si ça a l'air très pervers, d'une histoire pleine de cliffhangers. C'est-àdire que les événements se sont déroulés sur plusieurs jours et que personne, même pas les responsable, personne ne savait comment ça allait se terminer. Et en d'autres termes c'était bien sûr un peu en gros le must absolu, l'histoire absolue, absolue, absolument personne ne savait comment ça allait se terminer et tout le monde bien sûr était pris par le suspense : est-ce que ça allait bien finir ? Est-ce que ça allait mal finir ?'

Il nous semble illusoire de vouloir réduire la valeur des deux marqueurs ostensiblement métalinguistiques das heißt et mit anderen Worten à une affaire de choix des mots. L'équivalence entre une histoire pleine de cliffhangers et un déroulé des événements imprévisible non seulement pour les spectateurs mais aussi pour les protagonistes se tient : reste que le premier énoncé est suffisamment modalisé pour qu'il soit clair que l'affaire de Gladbeck n'était pas à proprement une telle "histoire ", ne serait-ce que 
parce qu'il ne s'agissait pas de fiction et qu'il n'y avait pas de scénariste, alors que l'énoncé en aval du marqueur, lui, réfère bien à l'affaire de Gladbeck et n'est pas modalisé ; on glisse donc de la comparaison à un jugement catégorique, en résorbant des différences auxquelles on faisait encore droit dans l'énoncé précédent. L'enchaînement suivant représente également un changement de point de vue qui va au-delà d'un habillage des choses par les mots qui laisserait le « contenu » inchangé : on bascule en effet de la question de la scénarisation du spectacle au domaine de la psychologie individuelle des spectateurs, via une prémisse tacite finalement assez contestable, à savoir que ce genre de "spectacle » fascine les gens - une proposition qu'il est d'autant plus fâcheux de traiter comme une prémisse qu'il s'agit en fait de la question initialement posée par le journaliste, et à laquelle l'invité n'a pas répondu. Si l'on ajoute à cela que ce glissement correspond aussi, sur le plan argumentatif, à un déplacement des responsabilités de l'industrie des médias vers les consommateurs, il faut au minimum parler d'un "usage stratégique » de la reformulation. Mais il nous semble plutôt que ces marqueurs sous-spécifiés excèdent le métalinguistique au sens strictement «formel » et correspondent à une opération plus générale d'identification d'un propos à un autre sur le plan du vouloir-dire, les deux propos articulés étant présentés comme deux façons différentes et inégalement adéquates ${ }^{8}$ de saisir le réel que l'on vise à dire. Ce faisant, on se rapproche de la thématique de la partialité du dire.

\section{Le cas de also}

Also est généralement classé comme un adverbe et glosé par "ainsi, donc». Il ne connaît aucune restriction de position et apparaît même fréquemment en position dite zéro, c'est-à-dire en tête d'énoncé, avant le premier constituant. Les formes licites dans cette position zéro sont généralement qualifiées de conjonctions de coordination. En l'espèce, il nous semble plutôt qu'il faille y voir un adverbial ayant la même fonction que lorsqu'il apparaît plus loin dans l'énoncé, mais qui exceptionnellement prend dans sa portée l'événement de la prise de parole. Un peu comme donc dans l'analyse qu'en propose Culioli (1990), also procède à une mise en relation de deux termes via identification ou implication, à tel point que l'on peut y voir y véritable connecteur, en tout cas pour certains de ses emplois. La valeur de consécution n'est qu'une interprétation possible en discours. L'exemple suivant porte sur la compatibilité entre transition écologique et économie de marché.

(12) Das ist eigentlich super, und je mehr Geld damit verdient werden kann, umso schneller würde es gehen. Das finde ich eigentlich nicht verwerflich. Schade finde ich, dass ä das Ganze so groß diskutiert wird, als sei es ökonomisch nicht machbar. Also der " Stern Report " hat damals ja errechnet, dass wir ein Prozent des Bruttosozialproduktes anders ausgeben müssen- ich sag gar nicht mal mehr ausgeben müssten-, um einen gefährlichen Klimawandel über die Zwei-Grad-Grenze hinaus zu vermeiden. Also die Umsteuerung, die wir jetzt vornehmen könnten in der Zeit, ist viel, viel geringer, als wenn wir warten, bis tatsächlich Klimawandel kommt. (DR 190913-02)

'En soi c'est super, et plus il y a d'argent à gagner, plus ça ira vite. En fait je ne trouve pas ça méprisable. Ce que je trouve dommage, c'est que euh on discute de tout cela comme si ça n'était pas faisable économiquement. Also le rapport Stern a calculé, à l'époque, qu'il fallait dépenser $1 \%$ du PNB autrement, je ne dis même pas «ne pas dépenser» pour éviter un changement climatique dangereux de plus de $2^{\circ}$. Also le changement de cap 
que nous pourrions entreprendre en ce moment est bien moindre que celui qui nous guette si nous attendons que le changement climatique s'installe vraiment.'

Dans ses emplois métalinguistiques, also présente les mêmes propriétés générales d'usage que les mots du dire de type A: il marque un retour au vouloir-dire avec disqualification tacite du dire antérieur, requalifié comme une première approche $d u$ nouveau dire, qui se caractérise par une plus grande adéquation au vouloir-dire.

(13) ... in eine Ortschaft, wo er auch FSA-, also Freie-Syrische-Armee-Bekannte hatte ... (DR 050913-05)

'... dans une localité où il avait également des connaissances au sein de la FSA, also l'Armée Syrienne Libre ...'

(14) Ich kann leider nicht Schule wechseln, also eigentlich schon, aber dann nur in die Hauptschule. (internet) ${ }^{9}$

'Malheureusement je peux pas changer d'école, also en fait si, mais alors seulement pour aller en école professionnelle.'

Also est licite en APP, et n'a pas besoin d'être en incise. Il y est la trace d'une opération assimilable à celle dont les mots du dire de type A sont l'indice, une relation sousspécifiée d'équivalence au niveau du "vouloir-dire ». Ainsi, dans l'exemple suivant, l'opération de retour du dire sur lui-même à des fins de réparation ne met pas véritablement en jeu le choix des mots. Il s'agit plutôt, après un détour ou une concession de rebrancher l'énonciation sur le propos le plus essentiel, qui entretient ici une relation de tension avec la proposition précédemment concédée. Le recours à also permet de se saisir de l'hétérogénéité des propos en co-présence pour faire entrer le propos concédé dans celui que l'on tente d'élaborer, nonobstant leur tension.

(15) [contexte : discussion sur les procédures de contrôle des exportations d'armes ; le locuteur, un parlementaire, se plaint du manque de coopération du «gouvernement fédéral». Son interlocuteur journaliste lui demande de confirmer que c'est bien le ministère de l'économie qui est responsable de ces dossiers.]

Das ist richtig, das Wirtschaftsministerium ist das federführende Ministerium, das im Grunde die Entscheidungen vorbereitet und das auch zum Beispiel die vorgesetzte Stelle ist für die Behörden, die jetzt zum Beispiel auch über die Chemiekomponenten-Lieferungen entschieden habendas ist in dem Fall das BAFA, das Bundesamt für Wirtschaft und ä Ausfuhrkontrolle. Und insgesamt also entscheidet aber die Bundesregierung dann gemeinsam über zum Beispiel die Waffenlieferungen, von denen Sie gerade gesprochen haben. (DR 200913-02)

'C'est juste, le ministère des finances est le ministère référent, qui au fond prépare les décisions et qui est aussi par exemple responsable des administrations qui ont la décision par exemple aussi sur les livraisons de composants chimiques, en l'occurrence c'est le BAFA, l'office fédéral pour l'économie et, euh, le contrôle de exportations. Et dans l'ensemble, also, par contre, c'est le gouvernement fédéral qui prend ensuite collectivement les décisions sur par exemple les livraisons d'armes dont vous avez parlé à l'instant.'

\begin{tabular}{|l|l|l|l|l|l|l|}
\hline Und & insgesamt & also & entscheidet & aber & die & Bundesregierung \\
\hline 'Et & dans l'ensemble & also & décide & mais & ART.DEF & gouvernement fédéral' \\
\hline
\end{tabular}


Un marqueur comme also est la trace d'une opération par laquelle l'énonciation est réamorcée et un nouveau point de vue élaboré préférentiellement à des tentatives précédentes d'approcher « ce qu'il y a à dire ». Ce mécanisme est très proche de celui qui préside à l'emploi des MD point de vue, et s'en distingue par un plus grand degré de " connectivité ", encore que certains contextes, notamment associés à une prise de contact ou à l'ouverture d'un nouveau tour de parole, ne présupposent aucune connexion entre les contenus articulés dans le cotexte amont. Les contextes où le réamorçage prend la forme d'une paraphrase métalinguistique correspondent simplement à une gamme d'emplois parmi d'autres.

\section{Conclusion}

Nous avons vu que les marqueurs du commentaire métalinguistique assimilables à des adverbes, en allemand, prennent place à l'intérieur d'un ensemble plus vaste de formes spécialisées dans le marquage de ce que nous avons appelé à la suite de Paillard «la partialité du dire ": marqueurs d'intégration linéaire, marqueurs à coloration plus dialogique ou concessive, exemplification... et enfin mots du dire, c'est-à-dire marqueurs métalinguistiques formés à partir d'une forme du verbe «dire » (sagen). La description linguistique des formes concernées, avec leurs similitudes et leurs différences, impose à nos yeux de reconnaître à la reformulation un rôle constitutif dans le processus d'énonciation. Les marqueurs métalinguistiques étudiés ici représentent une forme possible du marquage de cette « reformulativité » irréductible, mais n'en ont pas le monopole. À cet égard, ce bref tour d'horizon est aussi conçu comme un plaidoyer contre une séparation stricte entre ce qui relèverait strictement du métalinguistique et ce qui n'en relèverait pas.

\section{BIBLIOGRAPHIE}

Corpus et dictionnaires

Français :

Frantext. Corpus édité par l'UMR Analyse et traitement informatique de la langue française, Nancy.

http://www.atilf.fr/spip.php?article194 ou www.frantext.fr

Allemand:

Dereko: Institut für Deutsche Sprache (2014): Deutsches Referenzkorpus / Archiv der Korpora geschriebener Gegenwartssprache 2014-II (Release : 11.09.2014). Mannheim: Institut für Deutsche Sprache.

www.ids-mannheim.de/DeReKo 
DR : Modicom, P.-Y. (2013, rév. 2015). Deutschlandradio-Interviewkorpus 16.08.-23.09. 2013. Paris : Université Paris-Sorbonne.

GrWB : Institut für Deutsche Sprache (2004). GRAMMIS Grammatisches Wörterbuch, Mannheim: Institut für Deutsche Sprache.

http://hypermedia.ids-mannheim.de/call/public/gramwb.ansicht? $v_{-} a p p=g$

Bibliographie

Auchlin A. (1981). Réflexions sur les marqueurs de structuration de la conversation. Etudes de linguistique appliquée 44. 88-103.

Authier-Revuz J. (1984). Hétérogénéité(s) énonciative(s). Langages 73. 98-111.

Breindl E. (2008). Die Brigitte nun kann den Hans nicht ausstehen. Gebundene Topiks im Deutschen. Deutsche Sprache. 27-49.

Breindl E. (2011). Nach Rom freilich führen viele Wege. Zur Interaktion von Informationsstruktur, Diskursstruktur und Prosodie bei der Besetzung der Nacherstposition. In Ferraresi G. (éd.). Konnektoren im Deutschen. Tübingen : Narr. 1-40.

Breindl E., Volodina A., Waßner U. H. (2014). Handbuch der deutschen Konnektoren 2. Semantik der deutschen Satzverknüpfer. Berlin/New York : de Gruyter (= Schriften des Instituts für Deutsche Sprache 13.1-13.2).

Büring D. (2003). On D-Trees, Beans, and B-Accents. Linguistics \& Philosophy 26. 511-545.

Charolles M. (1997). L'encadrement du discours. Univers, champ, domaines et espaces. Cahiers de Recherche Linguistique LANDISCO 6. 1-73.

Culioli A. (1989(1990)). Donc. In Pour une Linguistique de l'énonciation I. Gap : Ophrys. 169-176.

Dalmas M. (2011). L'ouverture d'énoncé en allemand contemporain. Polycopié, université ParisSorbonne.

Espinat M. (2015). L'emploi d'unités polylexématiques idiomatiques à l'oral : Aspects linguistiques et psycho-cognitifs (corpus allemand et français). Thèse de doctorat, université Paris-Sorbonne.

Féry C. (2007). The prosody of topicalization. In Schwabe K. \& Winkler S. (eds). On Information Structure, Meaning and Form : Generalizations across langages. Amsterdam : John Benjamins. 69-86.

Franckel J.-J. \& Paillard D. (2008). Mots du discours: adéquation et point de vue. L'exemple de réellement, en réalité; en effet, effectivement. Estudos Linguisticos / Linguistic Studies 2. Lisbonne : Ediçoes Colibre/CLUNL. 255-274.

Frascarelli M. \& Hinterhölzl R. (2007). Types of topics in German and Italian. In Schwabe K. \& Winkler S. (eds). On Information Structure, Meaning and Form : Generalizations across langages. Amsterdam : John Benjamins. 87-116.

Khachatourian E. (2008). Les marqueurs de reformulation formés à partir du verbe dire. In Le Bot M.-C., Schuwer M. \& Richard E. (eds). La reformulation : marqueurs linguistiques, stratégies énonciatives. Rennes : PUR. 19-34.

Métrich R., Faucher E. \& Courdier G. (1998). Les invariables difficiles : dictionnaire allemand-français des particules, connecteurs, interjections et autres mots de la communication, tome I. Nancy : Nouveaux Cahiers d'Allemand.

Molnar V. (1998). Topic in focus: On the syntax, phonology, semantics and pragmatics of the socalled "contrastive topic" in Hungarian and German. Acta linguistica hungarica 45. 89-166. 
Murat M. \& Cartier-Bresson B. (1987). 'C'est-à-dire' ou la reprise interprétative. Langue française 73. 5-15.

Nølke H. (1993). Le Regard du Locuteur. Pour une linguistique des traces énonciatives. Paris : Kimé.

Paillard D. (2009). Prise en charge, commitment ou scène énonciative. Langue française 162.

109-128.

Pérennec M. (1982(2002)). Allerdings : pour un paradigme unique. In Pérennec M. Sur le texte. Énonciation et mots du discours en allemand. Lyon : PU Lyon. 125-141.

Reinhart T. (1981). Pragmatics and linguistics: An analysis of sentence topics in pragmatics and philosophy. Philosophica and Studia Philosophica Gandensia Ghent 27.1. 53-94.

Strawson P. F. (1964(1971)). Identifying reference and truth-values. In Strawson P.F. LogicoLinguistic Papers. Londres : Methuen and Co. 75-95.

Turco G. \& Coltier D. (1988). Des agents doubles de l'organisation textuelle, les marqueurs d'intégration linéaire. Pratiques 57. 57-79.

Zemb J.-M. (1978). Vergleichende Grammatik Französisch-Deutsch, I. Mannheim : Wissenschaftliche Buchgesellschaft / Dudenverlag.

\section{NOTES}

1. . Le terme est entendu ici dans un sens non-technique, sans allusion aux "grammaires de construction ".

2. . On entend par là que le constituant désigne une entité correspondant à " ce dont on parle " (topic) et construite en opposition plus ou moins explicite à autre chose dont on a parlé, dont on parlera, ou dont on aurait pu parler (« contrastif »).

3. . On se reportera notamment aux travaux de Jean-Marie Zemb, notamment Zemb (1978).

4. . Cf. Molnar (1998) pour l'équivalence des deux stratégies en allemand.

5. . Cette différence syntaxique nous semble lié au fait que tous ces marqueurs sont issus du figement de formes complexes encore facilement analysables et identifiables, et qui imposent leurs contraintes de construction. On peut ainsi montrer que das heißt, comme c'est-à-dire, impose la construction du segment en aval comme complément du verbe heißen, ce qui restreint l'éventail des constructions syntaxiques possibles à ce qu'il se trouve également être pour les conjonctions de coordination.

6. . http ://www.sueddeutsche.de/kultur/russland-und-fotograe-eigentlich-luegen-sienicht-1.327970

7. . Il convient de reconnaître que la frontière entre 2 et 4 reste assez poreuse.

8. . L'asymétrie bénéficie au segment en aval. Sur ce point, cf. déjà Murat \& Cartier-Bresson (1987).

9. . http ://www.kidsville.de/rettungsboot/forum/ich-bin/hilfe-mein-problem-alles/ 


\section{RÉSUMÉS}

Au sein des marqueurs discursifs de l'allemand, il est d'usage de distinguer une sous-classe de marqueurs métalinguistiques, dont certains incluent une forme du verbe «dire » (sagen) dans leur morphologie. L'objet de cet article est d'étudier la syntaxe et la sémantique de ces marqueurs à la fois dans le standard écrit et dans un corpus oral interactif. Une attention particulière est consacrée à la question de la position intermédiaire entre la première place et le verbe conjugué dans l'énoncé assertif, alors que l'allemand est réputé être une langue V2, car cette position intermédiaire semble réservée aux adverbiaux énonciatifs, dont les marqueurs métalinguistiques formés sur sagen. On compare ensuite ces adverbiaux métalinguistiques aux marqueurs présentant un comportement syntaxique de conjonction de coordination. Les adverbiaux métalinguistiques en sagen s'insèrent en fait dans un dispositif plus large de marqueurs énonciatifs. Ces observations conduisent à recentrer l'analyse sur la notion générale de reformulation, dont on montre qu'elle est liée à celle de point de vue.

Among German discourse markers, it is possible to identify a subset of lexicalized adverbials including the root of the verb 'to say' (sagen). The aim of this paper is to study the syntax and semantics of these metalinguistic adverbials both in written Standard German and in an oral, interactional corpus. Special attention is devoted to a special slot between the initial constituent of assertive utterances and the finite verb : while German is reported to be a V2 language, there seems to be a slot devoted to utterance-related adverbials, including metalinguistic sagenadverbials. Then, I compare these adverbials with other markers exhibiting the syntactic behaviour of conjunctions of coordination. Finally, metalinguistic adverbials appear to be part of a wider system of markers whose meaning crucially involves the notion of reformulation, which is shown to be intertwined with the question of 'viewpoint' in affirmations.

\section{INDEX}

Mots-clés : allemand, adverbes, coordination, reformulation, assertion

Keywords : German, adverbs, coordination, reformulation, assertion

\section{AUTEUR}

\section{PIERRE-YVES MODICOM}

Université Bordeaux-Montaigne (Equipe CLARE) 